\title{
Ehlers-Danlos Syndrome
}

${ }^{*}$ Mrs.Anitha.B

\section{Abstract:}

Ehlers-Danlos Syndrome (EDS) is a group of connective tissue disorders which are divided into various distinguishable phenotypes which is characterised by a collagen synthesis defect. The group of related conditions share a common decrease in the tensile strength and integrity of the skin, joints, and other connective tissues. The type of EDS determines the potential obstetric and medical complications. Major diagnostic criteria include joint hypermobility, skin involvement (Smooth and velvety), and absence of fragility or other significant skin or soft tissue abnormalities. There is a continuing need to expand awareness regarding optimal management for EDS hypermobility type relating to manage inherited disorder and prevent complications.

Key words: Ehlers-Danlos Syndrome, phenotypes, hyper mobility, obstetrics.

\section{INTRODUCTION}

Ehlers-Danlos Syndrome (EDS) is the name given to a group of more than 10 different inherited disorders, all involve a genetic defect in collagen and connective tissue synthesis and structure. Often involved parts are skin, joints, and blood vessel walls. Defects are caused by genetic mutations affecting collagen production

Ehlers-DanlosSyndrome can affect the skin, joints and blood vessels. This syndrome is clinically heterogenous, the underlying collagen abnormality is different for each type. Clinical recognition of the types of Ehlers-danlos syndrome is important. One type, type IV is associated with arterial rupture and visceral perforation, with possible life threatening consequences.

\section{Types}

- Classical Type (Types I \& II)

- Hypermobility Type (Type III)

- Vascular Type (Type IV)

- Kyphoscoliosis Type (Type VI)

- Arthrochalasia Type (Type VIIA/VIIB)

- Dermatosparaxis Type (Type VIIC)

\section{Classical Type (Types I \& II)}

$>$ Generalized hyperextensibility of joints and skin

$>$ Easy bruising, hemarthroses

$>$ Poor wound healing and retention of sutures

$>$ Congenital dislocation of hips

$>$ Scoliosis

$>M V P$

\section{Classical Type (Types I \& II)}

$>$ Generalized hyperextensibility of joints and skin

$>$ Easy bruising, hemarthroses

$>$ Poor wound healing and retention of sutures 
$>$ Congenital dislocation of hips

Scoliosis

MVP

\section{Hypermobility Type (Type III)}

- Most common type (1 in 10-15,000)

- Joint hyperextensibility

- Chronic degenerative joint disease

- Less skin involvement

- Advanced premature OA with pain

- MVP

\section{Vascular Type (Type IV)}

- Most serious type (1 in 250,000)

- Prone to ruptured/dissected arteries and aneurysms, intestinal and uterine rupture

- Easy bruising

- Visible veins beneath thin, translucent skin

- Protruding eyes, thin nose/lips, sunken cheeks, small chin

- Joint involvement variable

- Relative deficiency in type III collagen

\section{General symptoms}

- Stretchy skin

- Flexible joints with hypermobility

- Hernias

- Abnormal wound healing/stretching scars

- Joint dislocations

- Easy bruising

- Muscle weakness

- Heart and vascular problems such as aneurysms, MVP, aortic root dilatation

- Uterine and intestinal rupture

\section{Genetic inheritance}

- Primarily Autosomal Dominant

- Kyphoscoliosis and dermatosparaxis types are inherited in Autosomal Recessive pattern

\section{Diagnosis}

- Family History/Pedigree

- Physical Exam/History

- ECHO/DEXA

- Skin biopsy for vascular type

- Urine test for kyphoscoliosis type

- Genetic testing for classical, vascular, kyphoscoliosis and arthrochalasis types

- Can do prenatal and pre-implantation testing

\section{Complications}

- Scarring (molluscoidpseudotumors)

- Difficulty healing surgical wounds and sutures that tear out

- Chronic joint pain and joint dislocations

- Eye problems - globe rupture

- Premature rupture of membranes

- Rupture/dissection of major vessels or organs (uterine, intestinal, aortic)

\section{TREATMENT:}

- Managing symptoms and preventing complications

- Rehabilitation - aquatic therapy

- Patient education

- Body mechanics

- NSAIDs, chronic pain management

- Orthopedics and possible role for braces

- Antibiotic prophylaxis if MVP

- Minimize trauma and meticulous hemostasis during surgery

\section{REFERENCES:}

1. Klippel, John. Primer on the Rheumatic Diseases. Edition 12. Atlanta, GA: Arthritis Foundation; 2001: 584-586.

2. www.utdol.com

3. www.mayoclinic.com

4. Ramanath VS, Oh JK, Sundt III TM, Eagle KA. Acute Aortic Syndromes and Thoracic Aortic Aneurysm. Mayo Clinic Proceedings. 2009; 84(5):465-479. 